\title{
A UNIFORM ESTIMATE FOR THE RATE OF CONVERGENCE IN THE MULTIDIMENSIONAL CENTRAL LIMIT THEOREM FOR HOMOGENEOUS MARKOV CHAINS
}

\author{
M. GHARIB \\ Mathematics Department \\ Faculty of Science, Qatar University \\ STATE OF QATAR
}

(Received June 15, 1994 and in revised form January 17, 1995)

\begin{abstract}
In this paper a uniform estimate is obtained for the remainder term in the central limit theorem (CLT) for a sequence of random vectors forming a homogeneous Markov chain with arbitrary set of states. The result makes it possible to estimate the rate of convergence in the CLT without assuming the finiteness of the absolute third moment of the transition probabilities. Some consequences are also proved
\end{abstract}

KEY WORDS AND PHRASES. Uniform estimate, rate of convergence, Central limit theorem, homogeneous Markov chain.

1991 AMS SUBJECT CLASSIFICATION CODES. 60F05, 60J10.

\section{INTRODUCTION.}

Let $x_{1}, x_{2}, \ldots$ be a homogeneous Markov process with an arbitrary set of states $X$, defined by the transition probability function $P(\xi, A), \xi \in X, A \in \Im_{X}$ ( $\sigma$-algebra of subsets of $X$ ), and the initial distribution:

$$
P\left(x_{1} \in A\right)=\pi(A), \quad A \in \Im_{X}
$$

Suppose that $P(.,$.$) satisfies the following condition of uniform ergodicity:$

$$
\sup _{\xi, \eta \in X, A \in \Im_{X}}|P(\xi, A)-P(\eta, A)|=\rho<1 .
$$

It is well known that (see [13]) if (1.1) is fulfilled, then a stationary distribution $P(A), A \in \Im_{X}$ exists.

Let

$$
f(\xi)=\left(f_{1}(\xi), \ldots, f_{k}(\xi)\right),
$$

where $f_{\imath}(),.(i=\overline{1, k})$ are real $\Im_{X}$-measurable functions defined on $X$. Without loss of generality, in the next we shall suppose that

$$
\int_{\mathrm{X}} f_{\imath}(\eta) P(d \eta)=0, \quad i=\overline{1, k}
$$

Suppose that the matrix $\Lambda=\left\|\sigma_{\imath \jmath}\right\|_{1}^{k}$, where

$$
\sigma_{\imath j}=E_{p}\left[f_{\imath}\left(x_{1}\right) f_{j}\left(x_{1}\right)\right]+\sum_{r=1}^{\infty} E_{p}\left[f_{\imath}\left(x_{1}\right) f_{j}\left(x_{r+1}\right)\right]+\sum_{r=1}^{\infty} E_{p}\left[f_{j}\left(x_{1}\right) f_{\imath}\left(x_{r+1}\right)\right]
$$


is positive definite Let,

$$
\begin{gathered}
S_{n}=\frac{1}{\sqrt{n}} \sum_{r=1}^{n+1} B f\left(x_{r}\right), \quad P_{n}(A)=P\left(S_{n} \in A\right), \\
\triangle_{n}(A)=P_{n}(A)-\Phi(A), \quad A \in B^{k},
\end{gathered}
$$

where the matrix $B$ is such that $B^{\prime} B=\Lambda^{-1}, \Phi($.$) is the standard normal measure in \Re^{k}$, and $B^{k}$ is the class of all Borel sets in $\Re^{k}$.

In many articles (see for example, [2],[5],[6],[7], and [8]) uniform estimates (in particular, the Berry-Essen bounds) are obtained for the quantity:

$$
\sup _{A \in B_{0}^{k}}\left|\triangle_{n}(A)\right|,
$$

under the condition that the transition probability function $P(.,$.$) has a finite absolute moment of order$ not less than three. (Here $B_{0}^{k}$ is the class of all convex measurable sets in $\Re^{k}$ ).

In the present work a uniform estimate is obtained for (1.2) without assuming the finiteness of:

$$
m_{3}=\sup _{\xi \in X} \int_{X}|f(\eta)|^{3} P(\xi, d \eta) .
$$

This estimate extends the results pertaining to the case of the existence of absolute moments for the transition probabilities of order not less than the third for a sequence of random vectors that are either independent and identically distributed $(P(.,)=.P()=.\pi()$.$) , or linked in a homogeneous Markov$ chain (see, for example, [1], [19], and [20]).

Some other results, concerning (1.2), are also given.

We introduce the following "truncated" moments:

$$
\begin{aligned}
\beta_{n} & =\sup _{\xi \in X}\left[\int_{X_{n}}|f(\eta)|^{3} P(\xi, d \eta)+\sqrt{n} \int_{\bar{X}_{n}}|f(\eta)|^{2} P(\xi, d \eta)\right], \\
\delta_{n} & =\int_{X}|f(\xi)| P(d \xi) \int_{\bar{X}_{n}}\left|f(\eta)\left\|S(\xi, d \eta)\left|+\int_{\bar{X}_{n}}\right| f(\xi)\left|P(d \xi) \int_{X}\right| f(\eta)\right\| S(\xi, d \eta)\right|, \\
m_{\pi, 1} & =\int_{X_{n}}|f(\xi)| \pi(d \xi),
\end{aligned}
$$

and put,

$$
\begin{aligned}
& L_{\beta_{n}}=\frac{1}{\sqrt{n}} \sum_{r=1}^{k}\left[\lambda_{r}^{-3} \sup _{\xi \in X} \int_{X_{n}}\left|\left(\theta_{r}, f(\eta)\right)\right|^{3} P(\xi, d \eta)+\sqrt{n} \lambda_{r}^{-2} \sup _{\xi \in X} \int_{\bar{X}_{n}}\left|\left(\theta_{r}, f(\eta)\right)\right|^{2} P(\xi, d \eta)\right], \\
& L_{\delta_{n}}=\sum_{i=1}^{k} \sum_{j=1}^{k}\left(\lambda_{2} \lambda_{j}\right)^{-1}\left[\int_{X}\left|\left(\theta_{i}, f(\xi)\right)\right| P(d \xi) \int_{\bar{X}_{n}}\left|\left(\theta_{\jmath}, f(\eta)\right)\right||S(\xi, d \eta)|+\right. \\
& \left.+\int_{\bar{X}_{n}}\left|\left(\theta_{i}, f(\xi)\right)\right| P(d \xi) \int_{X}\left|\left(\theta_{j}, f(\eta)\right)\right||S(\xi, d \eta)|\right],
\end{aligned}
$$

and,

$$
L_{\pi, n}=\frac{1}{\sqrt{n}} \sum_{r=1}^{k} \lambda_{r}^{-1} \int_{X_{n}}\left|\left(\theta_{r}, f(\xi)\right)\right| \pi(d \xi),
$$

where $\theta_{\nu}, \nu=1,2, \ldots, k$ are orthonormal eigenvectors of $\Lambda$ and $\lambda_{\nu}^{2}$ are the corresponding eigenvalues,

$$
X_{n}=\{\xi:|f(\xi)| \leq \sqrt{n}\}, \quad \bar{X}_{n}=X \backslash X_{n},
$$

$|S(., A)|$ is the complete variation of the measure, 


$$
S(., A)=\sum_{r=1}^{\infty}\left(P^{r)}(., A)-P(A)\right), \quad A \in \Im_{X}
$$

and $P^{(r)}(.,$.$) is the transition probability function after r$-steps The measure $S(., A), A \in \Im_{X}$ exists in accordance with condition ( 11 1)

\section{FORMULATION OF THE RESULTS}

THEOREM 1. The inequality

$$
\sup _{A \in B_{0}^{k}}\left|\triangle_{n}(A)\right| \leq C(k, \rho)\left[L_{\beta_{n}}+L_{\delta_{n}}+L_{\pi, n}+\left(\frac{1}{3}+\frac{2}{3} \rho\right)^{n}\right],
$$

holds true. Where here and in what follows, $C($.$) denote a positive constant, not the same at different$ places, which depends only on the arguments in the parentheses

As it is shown in [1], [11], and [15] the expression $L_{\beta_{n}}$ characterizes the corresponding result in the case where summation of independent identically distributed random vectors $(p(.,)=.p()=.\pi()$.$) is$ considered. The expression $L_{\delta_{n}}$ characterizes, in some sense, the dependence structure of the random vectors

$$
f\left(x_{1}\right), \quad f\left(x_{2}\right), \quad \cdots
$$

( $L_{\delta_{n}}=0$ for independent identically distributed random vectors).

REMARK. If $g(x)$ is an increasing function, with positive argument, defined on $\Re^{k}$, such that $\frac{x}{g(x)}$ is non-decreasing, and $\lim _{x \rightarrow \infty} g(x)=\infty$, and if

$$
m_{g}=\sup _{\xi \in X} \int_{X}|f(\eta)|^{2} g(|f(\eta)|) P(\xi, d \eta)<\infty,
$$

then (2.1) remains true, but with the replacement of $\left(L_{\beta_{n}}+L_{\delta_{n}}\right)$ by $\left(L_{g}+Q_{g}\right) / g(k \sqrt{n})$, where

$$
\begin{aligned}
L_{g}=\sum_{r=1}^{k}\left(\lambda_{r}^{-3}+\lambda_{r}^{-2}\right) \sup _{\xi \in X} \int_{X} \mid\left(\theta_{r},\left.f(\eta)\right|^{2} g\left(\left|\left(\theta_{r}, f(\eta)\right)\right|\right) P(\xi, d \eta), \quad\right. \text { and } \\
Q_{g}=\sum_{i=1}^{k} \sum_{j=1}^{k}\left[\int_{X}\left|\left(\theta_{\imath}, f(\xi)\right)\right| P(d \xi) \int_{X}\left|\left(\theta_{\jmath}, f(\eta)\right)\right| g\left(\left|\left(\theta_{\jmath}, f(\eta)\right)\right|\right)|S(\xi, d \eta)|\right. \\
\left.+\int_{X}\left|\left(\theta_{\imath}, f(\xi)\right)\right| g\left(\left|\left(\theta_{\imath}, f(\xi)\right)\right|\right) P(d \xi) \int_{X}\left|\left(\theta_{\jmath}, f(\eta)\right)\right||S(\xi, d \eta)|\right] .
\end{aligned}
$$

If $\pi()=.p($.$) , then the sequence (2.2) forms a stationary sequence of random vectors and in this$ case Theorem 1 has the following simple form:

THEOREM 2. If $\pi()=.p($.$) , then$

$$
\sup _{A \in B_{0}^{k}}\left|\triangle_{n}(A)\right| \leq C(k, \rho)\left[L_{\beta_{n}}+L_{\delta_{n}}\right] .
$$

Repeating the arguments given in [3], and [4], one can see that the estimate (2.3) has a universal character. In particular, from (2.3) one can derive an analogy of the generalized Berry-Esseen inequality adduced in [12], and [16] for independent random vectors.

The next result follows from Theorem 1 .

THEOREM 3. If

$$
m_{2}=\sup _{\xi \in X} \int_{X}|f(\eta)|^{2} P(\xi, d \eta)<\infty
$$

then the CLT holds for the sequence (2.2), i.e. for any initial distribution $\pi($.$) :$ 


$$
\sup _{A \in B_{0}^{k}}\left|\triangle_{n}(A)\right| \rightarrow 0 \quad \text { as } \quad n \rightarrow \infty
$$

\section{AUXILIARY RESULTS}

Assume that

$$
\triangle=J
$$

where $J$ is the $k$-dimensional unit matrix Consequently $B=J$ Assume also that

$$
\frac{\beta_{n}}{\sqrt{n}}+\delta_{n} \leq C(k, \rho) \text {. }
$$

Through the proof of Theorem 1, it is shown that assumptions (3.1) and (3.2) do not bound the generality

Let

$$
\begin{gathered}
f_{n}(.)=\left(f_{n 1}(.), f_{n 2}(.), \ldots, f_{n k}(.)\right) \\
=\left[\begin{array}{cc}
f(.), & |f(.)| \leq \sqrt{n} \\
0, & |f(.)|>\sqrt{n}
\end{array}\right. \\
\bar{S}_{n}=\frac{1}{\sqrt{n}} \sum_{r=1}^{n+1} f_{n}\left(x_{r}\right), \quad \Psi_{n}(t)=E\left[e^{i\left(t . \bar{S}_{n}\right)}\right] .
\end{gathered}
$$

Put

$$
|\hat{t}|=\max (1,|t|), \quad \text { and } \quad T_{n}=\frac{\sqrt{n}}{50 \overline{\beta_{n}}}
$$

where

$$
\bar{\beta}_{n}=20 M^{3}(\rho) \beta_{n}, \quad \text { and } \quad M(\rho)=(9+12 \rho) /(1-\rho) .
$$

LEMMA 3.1. For $|t|<T_{n}$

$$
\begin{gathered}
\left|\Psi_{n}(t)-e^{-|t|^{2} / 2}\right| \leq C(k, \rho)\left[\left(\frac{\beta_{n}}{\sqrt{n}}+\delta_{n}\right) \max \left(|t|,|t|^{3}\right)+m_{\pi, 1} \frac{|t|}{\sqrt{n}}\right] e^{-t^{2} / 20} \\
+C(1) \beta_{n}\left(\frac{1}{3}+\frac{2}{3} \rho\right)^{n} \frac{|t|}{\sqrt{n}} .
\end{gathered}
$$

PROOF. Put

$$
\begin{aligned}
\sigma_{\imath \jmath}^{(n)}= & E_{p}\left[f_{n \imath}\left(x_{1}\right) f_{n \jmath}\left(x_{1}\right)\right]+\sum_{r=1}^{\infty} E_{p}\left[f_{n i}\left(x_{1}\right) f_{n \jmath}\left(x_{r+1}\right)-E_{p}\left[f_{n \imath}\left(x_{1}\right) f_{n \jmath}\left(x_{r+1}\right)\right]\right] \\
& +\sum_{r=1}^{\infty} E_{p}\left[f_{n \jmath}\left(x_{1}\right) f_{n \imath}\left(x_{r+1}\right)-E_{p}\left[f_{n \jmath}\left(x_{1}\right) f_{n \imath}\left(x_{r+1}\right)\right]\right] .
\end{aligned}
$$

It is clear that, for $i=\overline{1, k}, r=1,2, \ldots$

$$
E_{p}\left[f_{n i}\left(x_{1}\right)\right]=E_{p}\left[f_{n i}\left(x_{r+1}\right)\right] .
$$

Therefore,

$$
\left|E_{p}\left[f_{n 2}\left(x_{1}\right)\right]\right|=\left|\int_{\bar{X}_{n}} f_{\imath}(x) P(d x)\right| \leq \frac{\beta_{n}}{\sqrt{n}} .
$$


Similarly, it is easy to see that, for any $i, \jmath=\overline{1, k}$

$$
\left|E_{p}\left[f_{n \imath}\left(x_{1}\right) f_{n j}\left(x_{1}\right)\right]-E_{p}\left[f_{\imath}\left(x_{1}\right) f_{j}\left(x_{1}\right)\right]\right| \leq \frac{\beta_{n}}{\sqrt{n}} .
$$

Further, using ( 33 ), for any $i$ and $\jmath$

$$
\begin{aligned}
\mid E_{p}\left[f_{i}\left(x_{1}\right) f_{\jmath}\left(x_{r+1}\right)\right]-E_{p}[ & \left.f_{n \imath}\left(x_{1}\right) f_{n \jmath}\left(x_{r+1}\right)-E_{p}\left[f_{n \imath}\left(x_{1}\right)\right] E_{p}\left[f_{n \jmath}\left(x_{r+1}\right)\right]\right] \mid \\
\leq & \int_{X}|f(\xi)| P(d \xi) \int_{\bar{X}_{n}}|f(\eta)| V_{r}(\xi, d \eta) \\
& \quad \int_{\bar{X}_{n}}|f(\xi)| P(d \xi) \int_{X}|f(\eta)| V_{r}(\xi, d \eta)
\end{aligned}
$$

where $V_{r}(x,$.$) is the complete variation of the measure \left(P^{(r)}(x,)-.P().\right)$ From (3.4)-(3 6), it follows that

$$
\left|\sigma_{\imath \jmath}^{(n)}-\delta_{\imath \jmath}\right| \leq C(\rho)\left(\frac{\beta_{n}}{\sqrt{n}}+\delta_{n}\right)
$$

where $\delta_{\imath \jmath}$ is the kronker's symbol

Consider the $\Im_{X}$-measurable function

$$
f_{t, n}(x)=f_{t}(x)=\left(t, f_{n}(x)\right), \quad x \in X,
$$

and let $m_{t}=E_{p}\left[f_{t}\left(x_{1}\right)\right]$,

$$
\sigma_{t}^{2}=E_{p}\left[\left(f_{t}\left(x_{1}\right)-m_{t}\right)^{2}\right]+2 \sum_{r=1}^{\infty}\left[E_{p}\left[f_{t}\left(x_{1}\right) f_{t}\left(x_{r+1}\right)\right]-m_{t}^{2}\right]
$$

and

$$
\gamma_{t}^{3}=\sup _{\xi \in X} \int_{X}\left|f_{t}(\eta)-m_{t}\right|^{3} P(\xi, d \eta)
$$

It is easy to see that

$$
\left|m_{t}\right| \leq \sum_{\jmath=1}^{k}\left|t_{\jmath}\right|\left|E_{p}\left[f_{n \jmath}\left(x_{1}\right)\right]\right| \leq k|t| \frac{\beta_{n}}{n}
$$

and that

$$
\sigma_{t}^{2}=\left(t, \sigma_{n} t\right),
$$

where

$$
\sigma_{n}=\left\|\sigma_{\imath}^{(n)}\right\|_{1}^{k}
$$

It follows from (3.1) and (3.7)-(3.9) that,

$$
\left.\left.\left|\sigma_{t}^{2}-\right| t\right|^{2}\left|\leq C(k, \rho)\left(\frac{\beta_{n}}{\sqrt{n}}+\delta_{n}\right)\right| t\right|^{2},
$$

Consider now the sum of one-dimensional random variables

$$
\widehat{S}_{n}=\frac{1}{\sigma_{t} \sqrt{n}} \sum_{r=1}^{n+1}\left(f_{t}\left(x_{r}\right)-m_{t}\right) .
$$


and put

$$
\bar{\Psi}_{n}(\tau)=E\left(e^{2 \tau} \hat{S}_{n}\right), \quad \bar{T}_{n}=\frac{\sigma_{t}^{3} \sqrt{n}}{100 M^{3}(\rho) \gamma_{t}^{3}} .
$$

According to the results of [14], we have for $|\tau|<\bar{T}_{n}$,

$$
\left|\bar{\Psi}_{n}(\tau)-e^{-\frac{1}{2} \tau^{2}}\right| \leq\left[\frac{2 \gamma_{t}^{3}}{\sigma_{t}^{3} \sqrt{n}}\left(|\tau|^{3}+|\tau|\right)+\frac{m_{11}}{\sigma_{t} \sqrt{n}}|\tau|\right] e^{-\frac{1}{10} \tau^{2}}+\frac{2 \gamma_{t}^{3}}{\sigma_{t}^{3} \sqrt{n}}|\tau|\left(\frac{1}{3}+\frac{2}{3} \rho\right)^{n},
$$

where

$$
m_{11}=\int_{X}\left|f_{t}(\xi)\right| \pi(d \xi)
$$

In accordance with (3.2) and (3.10), we have

$$
\left.\left.\left|\sigma_{t}^{2}-\right| t\right|^{2}|\leq 1 / 2| t\right|^{2}
$$

and consequently,

$$
1 / 2 \leq \frac{\sigma_{t}^{2}}{|t|^{2}} \leq 3 / 2
$$

As a consequence of (3.8), it is easy to see that

$$
\gamma_{t}^{3} \leq 5|t|^{3} \beta_{n} .
$$

On putting (see [14]) $\bar{\beta}_{n}=20 M^{3}(\rho) \beta_{n}$, we get from (3.12) and (3.13), that

$$
\frac{\sigma_{t}}{\bar{T}_{n}}=\frac{100 M^{3}(\rho) \gamma_{t}^{3}}{\sigma_{t}^{2} \sqrt{n}} \leq \frac{50 \bar{\beta}_{n}|t|}{\sqrt{n}} \leq 1, \quad \text { for } \quad|t| \leq \frac{\sqrt{n}}{50 \bar{\beta}_{n}}=T_{n}
$$

It is not hard to see that

$$
\Psi_{n}(t)=e^{i \sqrt{n} m_{t}} \bar{\Psi}_{n}\left(\sigma_{t}\right) .
$$

From (3.11) and (3.14), on noticing that $m_{11} \leq|t| m_{\pi, 1}$, and $\gamma_{t}^{3} / \sigma_{t}^{3} \leq 10 \sqrt{2}$, it follows that

$$
\left|\Psi_{n}(t)-e^{i \sqrt{n} m_{t}-\frac{1}{2} \sigma_{t}^{2}}\right| \leq\left[50 \sqrt{3} \frac{\beta_{n}}{\sqrt{n}} \mid \widehat{t}^{3}+\frac{|t|}{\sqrt{n}} m_{\pi, 1}\right] e^{-\frac{1}{20}|t|^{2}}+20 \frac{\beta_{n}}{\sqrt{n}}|t|\left(\frac{1}{3}+\frac{2}{3} \rho\right)^{n}
$$

Further, using (3.8), (3.10), and (3.12), consistent with (3.2), it is easy to see that

$$
\left|e^{i \sqrt{n} m_{t}-\frac{1}{2} \sigma_{t}^{2}}-e^{-\frac{1}{2}|t|^{2}}\right| \leq\left[K|t| \frac{\beta_{n}}{\sqrt{n}}+C(k, \rho)\left(\frac{\beta_{n}}{\sqrt{n}}+\delta_{n}\right)|t|^{2}\right] e^{-\frac{1}{4}|t|^{2}} .
$$

Finally, the assertion of the lemma follows from (3.15) and (3.16).

Through the proof of Theorem 1, we shall need estimates for the derivatives of the characteristic function $\Psi_{n}($.$) . Let us fix a direction \theta$ and let $t_{\theta}$ be the projection onto $\theta$.

LEMMA 3.2. a. For any $\nu \geq 0$, and for $|t| \leq C_{0}(k, \rho) T_{n}^{1 / 2}$

$$
\sup _{\theta}\left|\frac{\partial^{\nu}}{\partial t_{\theta}^{\nu}}\left(\Psi_{n}(t)-e^{-\frac{1}{2}|t|^{2}}\right)\right| \leq C(k, \rho, \nu)\left[|\widehat{t}|^{\nu^{*}} e^{-\frac{1}{20}|t|^{2}}\left(\frac{\beta_{n}}{\sqrt{n}}+\frac{m_{\pi, 1}}{\sqrt{n}}\right)+\frac{m_{1}}{\sqrt{n}}(1 / 3+2 / 3 \rho)^{n}|t|\right]
$$

where 


$$
\nu^{*}=\max (2 \nu, \nu+3), \quad m_{1}=\sup _{\xi \in X} \int_{X}|f(\eta)| P(\xi, d \eta)
$$

b For any $\nu>0$, and for $|t| \leq T_{n}$

$$
\sup _{\theta}\left|\frac{\partial^{\nu}}{\partial t_{\theta}^{\nu}} \Psi_{n}(t)\right| \leq c(k, \rho, \nu)\left[|\widehat{t}|^{\nu} e^{-\frac{1}{20}|t|^{2}}+\frac{m_{1}}{\sqrt{n}}|t|\left(\frac{1}{3}+\frac{2}{3} \rho\right)^{n}\right] \text {. }
$$

The proof of this lemma is easily obtained by repeating, word for word, the arguments of the proof of Lemma 21 in [8] with the use of the general spectral theory of linear operators acting in the Banach space of all bounded functions with the uniform norm Through the proof of part $b$, Lemma 3 of Chapter 6 in [16] is used

Let

$$
\triangle(A)=\Phi(A)-P(A), \quad A \in B^{k},
$$

where $P($.$) is a distribution function in \Re^{k}$. Let $\eta^{(1)}$ be such a vector that

$$
P_{1}^{\prime}(A)=P\left(\eta^{(1)} \in A\right), \quad \text { and } \quad P\left(\left|\eta^{(1)}\right|>h\right) \leq \chi_{1} h^{-1},
$$

where $\chi_{1}=\chi_{1}(k)$ depends only on $k$.

For any $T>0$, put

$$
\eta^{(T)}=T^{-1} \eta, \quad P_{T}^{\prime}(A)=P\left(\eta^{(T)} \in A\right), \quad \text { and } \quad \triangle_{T}(A)=\left(\triangle * P_{T}^{\prime}\right)(A)
$$

where $*$ denotes the composition operation.

The following lemma is proved in [18] and [20].

LEMMA 3.3. For any $T>0$

$$
\sup _{A \in B_{0}^{k}}\left|\triangle_{n}(A)\right| \leq 2 \sup _{A \in B^{k}}\left|\triangle_{T}(A)\right|+\frac{12 \sqrt{2}}{\sqrt{2 \pi}} \frac{\chi_{1}}{T} k^{3 / 2} .
$$

If $\eta^{(1)}$ has the distribution with density

$$
u(x)=\alpha_{k, r}\left[J_{k / 2}(|x| / 2 r) /|x|^{k / 2}\right]^{2 r}
$$

where $J_{k / 2}($.$) is the Bessel function of order k / 2$, the constant $\alpha_{k, r}$ is such that $\int_{\mathfrak{P}^{k}} u(x) d x=1$, and $r$ is a positive integer, then for all $\nu \leq k+q$

$$
E\left[\left|\eta^{(1)}\right|^{\nu}\right] \leq \chi_{\nu}(k, q)<\infty, \quad \text { if } \quad r>2+\frac{q-1}{k+1}
$$

and for $|t|>1$

$$
\int_{\Re^{k}} e^{z(t, x)} P_{1}^{\prime}(d x)=0
$$

\section{PROOFS OF THE THEOREMS}

PROOF OF THEOREM 1. Let

$$
\bar{P}_{n}(A)=P\left(\bar{S}_{n} \in A\right), \quad \text { and } \quad \bar{\triangle}_{n}(A)=\bar{P}_{n}(A)-\Phi(A), \quad A \in B^{k} .
$$

It is not difficult to see that for any $A \in B^{k}$

$$
\left|P_{n}(A)-\bar{P}_{n}(A)\right| \leq \sum_{r=1}^{n+1} P\left(\left|f\left(x_{r}\right)\right|>\sqrt{n}\right) .
$$


Further

$$
P\left(\left|f\left(x_{1}\right)\right| \geq \sqrt{n}\right)=\int_{\bar{X}_{n}} \pi(d x) \leq \frac{m_{\pi, 1}}{\sqrt{n}}
$$

and for $r \geq 2$

$$
\begin{aligned}
P\left(\left|f\left(x_{r}\right)\right| \geq \sqrt{n}\right)= & \int_{X} P^{(r-1)}\left(x, \bar{X}_{n}\right) \pi(d x) \leq \sup _{x} P^{(r-1)}\left(x, \bar{X}_{n}\right) \leq \sup _{x} P\left(x, \bar{X}_{n}\right) \\
& \leq \frac{1}{(\sqrt{n})^{3}} \sqrt{n} \sup _{\xi \in X} \int_{\bar{X}_{n}}|f(\eta)|^{2} P(\xi, d \eta) \leq \frac{\beta_{n}}{(\sqrt{n})^{3}} .
\end{aligned}
$$

It follows from (4 1)-(4 3) that

$$
\left|P_{n}(A)-\bar{P}_{n}(A)\right| \leq \frac{m_{\pi, 1}}{\sqrt{n}}+\frac{\beta_{n}}{\sqrt{n}}
$$

Therefore, for any $A \in B^{k}$

$$
\left|\triangle_{n}(A)\right| \leq\left|\bar{\triangle}_{n}(A)\right|+\frac{m_{\pi, 1}}{\sqrt{n}}+\frac{\beta_{n}}{\sqrt{n}} .
$$

Let $T>0$ and $\bar{\triangle}_{n, T}(A)=\left(\bar{\triangle}_{n} * P_{T}^{\prime}\right)(A)$, where $P_{T}^{\prime}$ is the distribution with the density defined by (3.17). It follows from Lemma 3.3 that

$$
\sup _{A \in B_{0}^{k}}\left|\bar{\triangle}_{n}(A)\right| \leq 2 \sup _{A \in B^{k}}\left|\bar{\triangle}_{n, T}(A)\right|+\frac{C(k)}{T} .
$$

Let

$$
V_{T}(t)=E\left[e^{i\left(t, \eta^{(T)}\right)}\right]
$$

Thus, from (3.18) and (3.19), we have $V_{T}(t)=0$, for $|t|>T$, and for all $\nu \leq k+q$

$$
\sup _{\theta}\left|\frac{\partial^{\nu}}{\partial t_{\theta}^{\nu}} V_{T}(t)\right| \leq E\left[\left|\eta^{(T)}\right|^{\nu}\right] \leq \frac{C(k, \nu)}{T^{\nu}} .
$$

Now, we put $T=T_{n}$ (defined in Lemma 3.1), and $q=1$. It is clear that the generalized measure $\bar{\triangle}_{n, T_{n}}$ with the characteristic function

$$
W_{n}(t)=\left(\Psi_{n}(t)-e^{-\frac{1}{2}|t|^{2}}\right) V_{T_{n}}(t)
$$

satisfies all the conditions of Lemma 1 in [17]. Hence, from (4.7), for any $0<\nu_{0}<\infty$, we have

$$
\int_{\Re^{k}}\left|\frac{\partial^{\nu_{0}}}{\partial t_{\theta}^{\nu_{\theta}}} W_{n}(t)\right| d t \leq C\left(T_{n}, \nu_{0}\right) \max _{\nu=\overline{0, \nu_{0}}} \int_{|t| \leq T_{n}}\left|\frac{\partial^{\nu}}{\partial t_{\theta}^{\nu}}\left(\Psi_{n}(t)-e^{-\frac{1}{2}|t|^{2}}\right)\right| d t
$$

where

$$
C\left(T_{n}, \nu_{0}\right)=\sum_{\nu=0}^{\nu_{0}}\left(\begin{array}{c}
\nu \\
\nu_{0}
\end{array}\right) C(\nu, k) / T_{n}^{\nu}
$$

In accordance with (3.2) it follows from Lemmas 3.1 and 3.2, that for any $\nu \leq k+1$

$$
\sup _{\theta} \int_{|t| \leq T_{n}}\left|\frac{\partial^{\nu}}{\partial t_{\theta}^{\nu}}\left(\Psi_{n}(t)-e^{-\frac{1}{2}|t|^{2}}\right)\right| d t \leq C(k, \rho, \nu)\left[\frac{\beta_{n}}{\sqrt{n}}+\delta_{n}+\frac{m_{\pi, 1}}{\sqrt{n}}+\left(\frac{1}{3}+\frac{2}{3} \rho\right)^{n}\right] .
$$

It follows from (4.9), on using Lemma 1 of [17], that 


$$
\sup _{A \in B^{k}}\left|\bar{\triangle}_{n, T_{n}}(A)\right| \leq C(k, \rho)\left[\frac{\beta_{n}}{\sqrt{n}}+\delta_{n}+\frac{m_{\pi, 1}}{\sqrt{n}}+\left(\frac{1}{2}+\frac{2}{3} \rho\right)^{n}\right] .
$$

Theorem 1 follows from (4 5), (4 6), and (4 10) if conditions ( 31 1) and (3 2) are satisfied

It is clear that the generality of Theorem 1 is not lost by condition $\left(\begin{array}{l}3 \\ 2\end{array}\right)$ because in the opposite

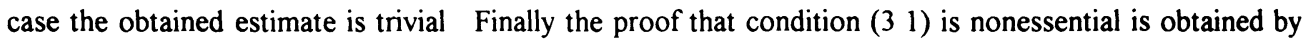
repeating, word-for-word, the corresponding part in the proof of Theorem 31, p. 108 in [19].

PROOF OF THEOREM 2. The proof of Theorem 2 goes along the lines of the proof of Theorem 1, where the condition $\pi()=.P($.$) is taken into account.$

PROOF OF THEOREM 3. It suffices to prove Theorem 3 under the condition $\pi()=.P($.) (the general case reduces to this partial one) By Theorem 1 it suffices to show that

$$
L_{\beta_{n}}+L_{\delta_{n}} \rightarrow 0 \quad \text { as } \quad n \rightarrow \infty \text {. }
$$

Since $m_{2}<\infty$, then

$$
\sup _{\xi \in X} \int_{\bar{X}_{n}}\left|\left(\theta_{r}, f(\eta)\right)\right|^{2} P(\xi, d \eta) \rightarrow 0, \quad \text { as } \quad n \rightarrow \infty .
$$

It is not hard to see that

$$
\begin{aligned}
\frac{1}{\sqrt{n}} \int_{X_{n}}\left|\left(\theta_{r}, f(\eta)\right)\right|^{3} P(\xi, d \eta) \leq C(k) & {\left[\frac{1}{\sqrt[4]{n}} \int_{\bar{X}_{n}}\left|\left(\theta_{r}, f(\eta)\right)\right|^{2} P(\xi, d \eta)\right.} \\
+ & \left.\int_{\{\eta|f(\eta)|>\sqrt[4]{n}\}}\left|\left(\theta_{r}, f(\eta)\right)\right|^{2} P(\xi, d \eta)\right] .
\end{aligned}
$$

Hence,

$$
L_{\beta_{n}} \rightarrow 0, \quad \text { as } \quad n \rightarrow \infty .
$$

Also, for $L_{\delta_{n}}$, one can show, using Holder's inequality, that

$$
\begin{gathered}
L_{\delta_{n}} \leq \frac{4}{1-\rho} \sum_{i=1}^{k} \sum_{\jmath=1}^{k}\left\{\left[\int_{X} \mid\left(\theta_{i},\left.f(\xi)\right|^{2} P(d \xi)\right]^{1 / 2}\left[\int_{\bar{X}_{n}}\left|\left(\theta_{\jmath}, f(\eta)\right)\right|^{2} P(\xi, d \eta)\right]^{1 / 2}\right.\right. \\
+\left[\int_{\bar{X}_{n}}\left|\left(\theta_{\imath}, f(\xi)\right)\right|^{2} P(d \xi)\right]^{1 / 2}\left[\int_{X} \mid\left(\theta_{\jmath},\left.f(\eta)\right|^{2} P(\xi, d \eta)\right]^{1 / 2}\right\} .
\end{gathered}
$$

It is clear from (4.13) that

$$
L_{\delta_{n}} \rightarrow 0, \quad \text { as } \quad n \rightarrow \infty .
$$

Finally, from (4.12) and (4.14), we get the assertion of Theorem 3.

\section{REFERENCES}

[1] BHATTACHARYA, R. N., Berry-Esseen bounds for the multidimensional central limit theorem, Bull. Amer. Math. Soc., 74 (1968), 285-287.

[2] BOLTHAUSEN, E., The Berry-Esseen theorem for strongly mixing Harris recurrent Markov chains, Z. Wahrscheinlichkeitstheorie und ver. Geb., 60, 3 (1982), 283-289.

[3] ESSEEN, C. G., On the remainder term in the central limit theorem, Arkiv. Math., 81 (1969), 7 15.

[4] FELLER, W., On the Berry-Esseen theorem, Z. Wahrscheinlichkeits Theorie und ver. Geb., 3 (1968), 261-268. 
[5] FORMANOV, $\mathrm{SH} \mathrm{K}$, On the rate of convergence in the multidimensional limit theorem for homogeneous Markov chains, Dokl. Akad. Nauk SSSR, 2041 (1972), 29-31

[6] FORMANOV, SH K, A uniform estimate in the multidimensional limit theorem for homogeneous Markov chains on the class of all convex measurable sets I., Izd-vo AN IzSSR, Phys.-Math. Ser., 3 (1972), 33-37

[7] FORMANOV, SH K, A uniform estimate in the multidimensional limit theorem for homogeneous Markov chains on the class of all convex measurable sets II, Izd-vo AN UzSSR, Phys.-Math. Ser., 6 (1972), 35-42

[8] FORMANOV, SH K, Some estimates of the rate of convergence in the multidimensional limit theorems for homogeneous Markov processes, Lecture Notes in Math., 550 (1976), 80-98

[9] FORMANOV, SH K, On uniform estimates of the remainder term in the multidimensional limit theorem for homogeneous Markov chains I, Izd-vo AN UzSSR, Phys.-Math. Ser., 3 (1971), 24-30

[10] FORMANOV, SH K, On uniform estimates of the remainder term in the multidimensional limit theorem for homogeneous Markov chains II, Izd-vo AN UzSSR, Phys.-Math. Ser., 5 (1971), 20-25

[11] IBRAGIMOV, I A and OSIPOV, L V., On an estimate of the remainder term in Lindeberg's theorem, Theory Prob. Appl., 11 (1966), 125-128

[12] KATZ, M L., Note on the Berry-Esseen theorem, Ann. Math. Statist., 34 (1963), 1107-1 108

[13] NAGAEV, S. V., Some limit theorems for stationary Markov chains, Theory Prob. Appl., 2 (1957), 378-406.

[14] NAGAEV, S. V., More exact statements of limit theorems for homogeneous Markov chains, Theory Prob. Appl., 6 (1961), 62-81.

[15] PETROV, V. V. and OSIPOV, L. V., On estimates of the remainder term in the central limit theorem, Theory Prob. Appl., 12, 2 (1967), 322-329.

[16] PETROV, V. V., Sums of Independent Random Variables, Springer-Verlag, 1975

[17] ROTAR, V. I., A non-uniform estimate for the rate of convergence in the multidimensional central limit theorem, Theory Prob. Appl., 15, 4 (1970), 630-648.

[18] SAZONOV, V. V., On the multidimensional central limit theorem, Sankhya, Ser. A, 30 (1968), 203-225.

[19] SIRAZHDINOV, S. KH. and FORMANOV, SH K., Limit theorems for sums of random vectors connected in a homogeneous Markov chain, Fan, Tashkent. (1979) (In Russian).

[20] VON BAHR, B., Multidimensional integral limit theorem, Arkiv. Math., 7, 6 (1967), 71-88. 


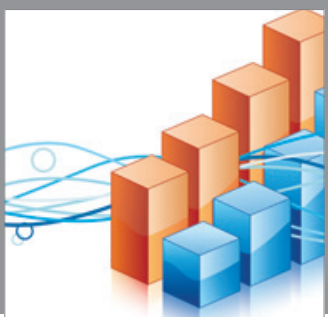

Advances in

Operations Research

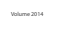

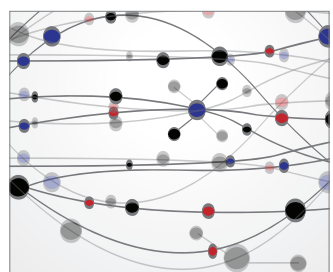

\section{The Scientific} World Journal
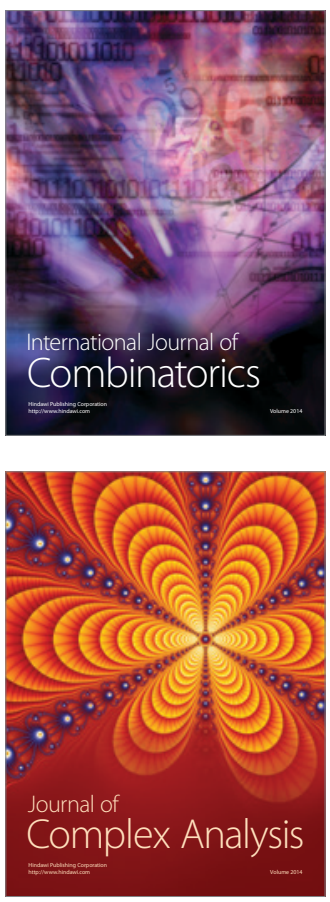

International Journal of

Mathematics and

Mathematical

Sciences
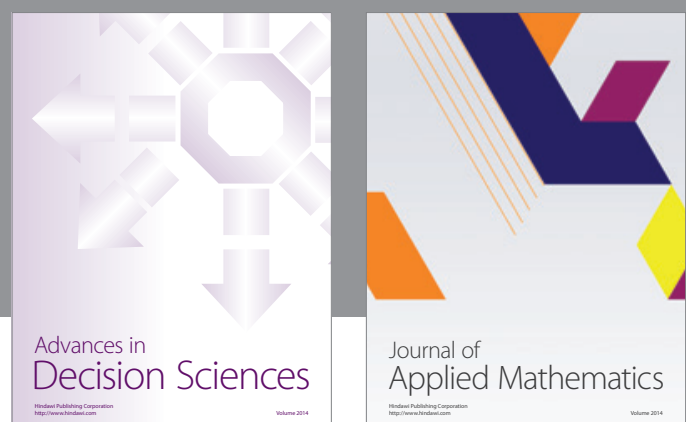

Journal of

Applied Mathematics
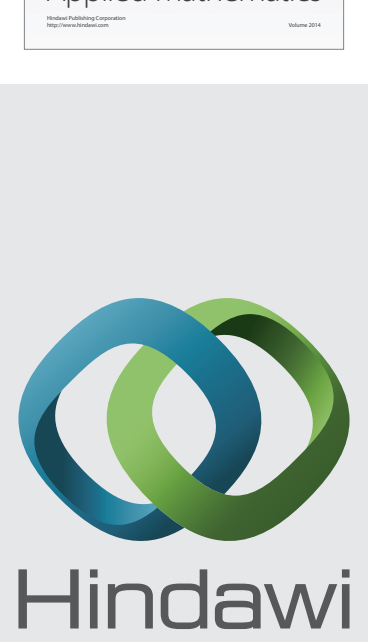

Submit your manuscripts at http://www.hindawi.com
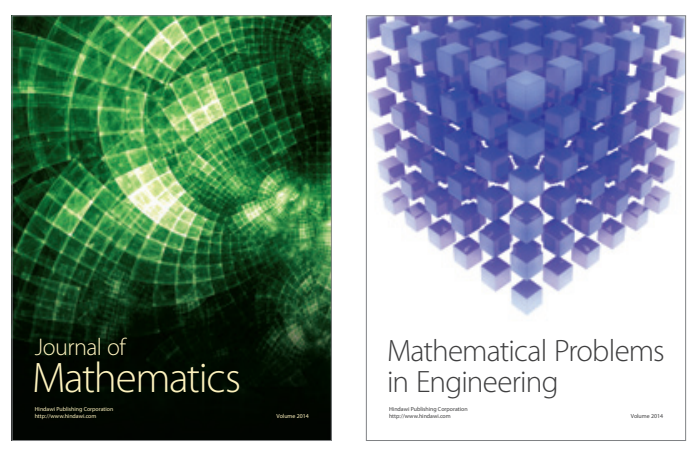

Mathematical Problems in Engineering
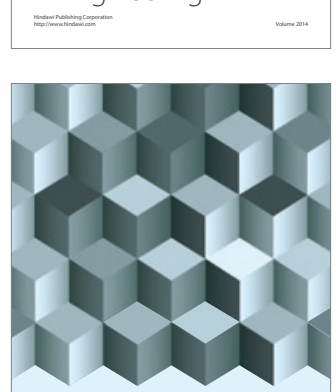

Journal of

Function Spaces
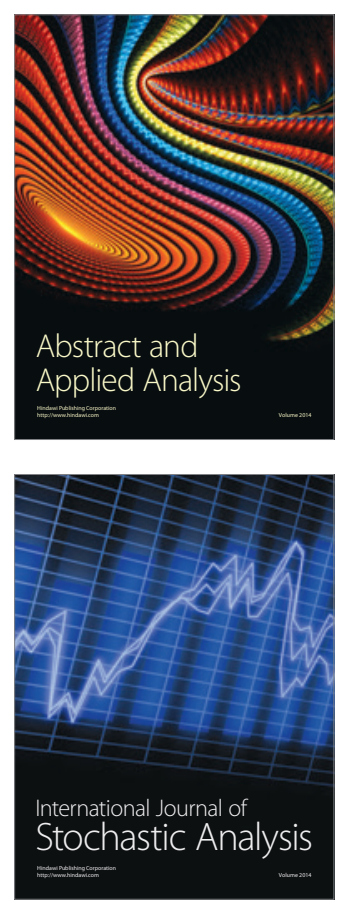

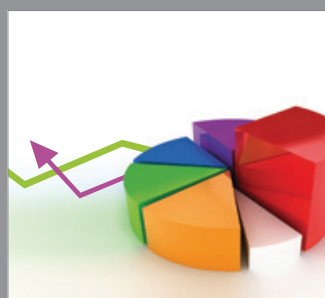

ournal of

Probability and Statistics

Promensencen
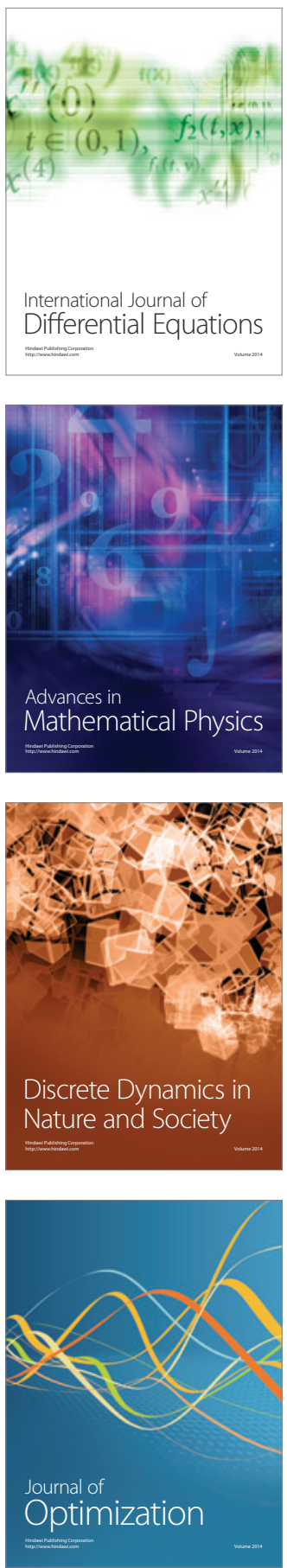